\title{
Echocardiographic Evaluation of Females with Asthma
}

\author{
Gokhan Perincek \\ Kars Harakani State Hospital, Department of Respiratory Diseases, Kars, Turkey \\ Email: md.gokhanperincek@gmail.com \\ Timor Omar \\ Kars Harakani State Hospital, Department of Cardiology, Kars, Turkey \\ Email: tbigmurad@gmail.com \\ Sema Avci (Corresponding author) \\ Amasya University Sabuncuoglu Serefeddin Research and Training Hospital, \\ Department of Emergency Medicine, Amasya, Turkey \\ Email: dnzlsema@gmail.com
}

\begin{abstract}
Objective: To evaluate ventricular functions and atrial conduction times in females with mild-tomoderate asthma

Design: Prospective cohort study

Place \& duration of the study: Kars Harakani State Hospital, Department of Respiratory Diseases, March to August 2019

Methodology: One hundred and sixteen patients with mild-to-moderate asthma and fifty healthy subjects enrolled. Basic characteristics, blood variables and echocardiographic parameters were compared between two groups.

Results: CRP ( $\mathrm{p}=0.017)$, CAR $(\mathrm{p}=0.01)$, eosinophil count $(\mathrm{p}=0.001)$ were higher and albumin $(\mathrm{p}=0.001)$ was lower in the asthma group. End-systolic diameter $(\mathrm{p}=0.029)$, interventricular septum thickness $(\mathrm{p}=0.022)$ and posterior wall thickness $(\mathrm{p}=0.027)$ were higher in the group with asthma. Emax $(\mathrm{p}=0.040)$ and E/A mitral ( $\mathrm{p}=0.040)$ decreased in asthmatic females. Aortic diameters consist of sinus $(\mathrm{p}=0.041)$, sinutubular junction $(\mathrm{p}=0.037)$ and ascending aorta $(\mathrm{p}=0.006)$ increased significantly and lateral/tricuspid AEMD prolonged $(\mathrm{p}=0.038)$ in the patients with asthma. In the asthma group, RV free wall thickness was higher $(\mathrm{p}=0.043)$.
\end{abstract}

Conclusion: LV end-systolic diameter, wall thickness, diastolic functions, aortic diameters, lateral/tricuspid AEMD and RV free wall thickness were affected by asthma in females.

Key words: asthma, females, echocardiography

Special Issue of Health Sciences

DOI: $10.7176 / J S T R / 6-03-06$

\section{Introduction}

Asthma is a common chronic inflammatory disorder of the airways which is related to completely or partially reversed airflow obstruction, hyper-responsiveness and represented recurrent dyspnea, wheezing and coughing. ${ }^{1,2}$ Asthma is a significant public health problem leading to morbidity, mortality, and impaired quality of life totally, affecting people of all genders, races, and age groups. ${ }^{3}$ Asthma disease may affect several organs including the heart. ${ }^{2}$ 
In asthma, chronic inflammation cause to release of inflammatory mediators in the lung, these mediators directly change the vascular endothelium, on the other hand, induce indirect systemic inflammation through the liver. This systemic inflammation causes procoagulation, which prepare the ground for cardiovascular disorders. ${ }^{4}$ Severe and mild-to-moderate asthma may have different levels of inflammation and inflammatory mediators, as well as the level of cardiac functions and vascular structures may be affected by the severity of asthma.

In several studies, ventricular systolic and diastolic function changes in asthmatic patients including different stages have been shown. ${ }^{1,2,5,6}$ In the study we compared blood variables including inflammatory markers, ventricular functions and atrial conduction times in female patients with mild-to-moderate asthma and healthy controls by using conventional echocardiography and tissue Doppler imaging (TDI).

\section{Methodology \\ Design and Assessment}

This prospective cohort study was conducted with the approval of Kafkas University Medical Faculty, Kars, Turkey ethics committee from March to August 2019. Female patients with mild to moderate asthma were enrolled in the study. Asthma severity was determined according to Global Initiative for Asthma Management and Prevention. ${ }^{7}$ The control group consisted of adult females conceived healthy. The group with asthma comprised of females with clinical and spirometric diagnosis of asthma, for more than six weeks with no history of acute upper or lower exacerbation of the disease. The control group had a normal lung functions.

Females with chronic obstructive pulmonary disease, asthma exacerbation, pulmonary embolism, valvular heart disease, wall-motion abnormality, irregular blood pressure, insulin dependent diabetes mellitus, patients using two or more oral antidiabetic drugs, hypo-hyperthyroidism, anemia, renal impairment, lipid abnormalities, coronary artery disease, heart failure, structural heart diseases, atrioventricular conduction abnormalities, prior use of antiarrhythmic drugs, ejection fraction $<50 \%$, malignancy, pneumonia, electrolyte disorders, patients who fail to comply echocardiography.

Age, smoking status, biomass exposure, inflammatory markers including c-reactive protein (CRP), creactive protein/albumin ratio (CAR), neutrophil/lymphocyte ratio (NLR), platelet/lymphocyte ratio (PLR), pulmonary function tests and transthoracic echocardioraphy were conducted. Forced expiratory volume in the first second (FEV1) and forced vital capacity (FVC) were measured at baseline using a spirometer (Spirolab III-MIR, Italy).

\section{Blood samples}

All blood samples were drawn from the vein in the forearm and collected into $2 \mathrm{~mL}$ Lavender (EDTA) top tube $+4 \mathrm{~mL}$ Yellow (Acid Citrate Dextrose) top tube. The samples were analysed with Pentra DF Nexus, Horiba Medical, Japan with Automated Cell Counter Methodology and Cobas C 702 Module, Roche, Switzerland. The complete blood count samples were stabilized optimally when run within in 4 hours of collection, stable for 24 hours at room temperature, and stable for 36 hours at $2-8$ degrees C. The yellow top tube was centrifuged for 8-10 minutes at 3500-4000 revolution per minute (rpm) and serum was seperated. The serum for albumin and CRP was stable for 8 hours at 2-8 degrees C.

\section{Echocardiograhy}

Transthoracic echocardiography (Epiq 7; Philips) was evaluated by the same practioner in a standard protocol in all patients. Patients were monitored using electrocardiographic leads and were placed in the left lateral decubitus position. Echocardiographic images were obtained from the parasternal views (long axis, short axis), the apical 4-chamber view and, the subcostal view. Echocardiographic measurements were performed at the end of expiration according to the recommendations of the American Society of Echocardiography/European Association of Echocardiography. ${ }^{8}$

- Diameters of right ventricle (RV) and wall thickness were measured in apical and subcostal views.

- Left ventricle (LV) diameter and wall thickness were measured in the parasternal view.

- Left atrial diameter, measured in the parasternal view.

- Aortic root diameters, measured at the sinuse of Valsalva.

- Left ventricle ejection fraction (LVEF), measured in apical 4-chamber view by modified Simpson method.

- LV functions were evaluated as follows: a) maximal peak velocity of early diastolic flow (Emax) and maximal peak velocity of atrial contraction (Amax), measured over the mitral valve. 
b) E/A demonstrates the ratio of peak velocity blood flow from gravity in early diastole (E) to peak velocity flow in the late diastole originated from atrial contraction (A) with TDI.

c) early diastolic mitral annulus velocity (e') measured by TDI and the ratio of the transmitral early peak velocity (E) by pulsed wave Doppler over e' (E/e').

- RV functions were evaluated as follows:

a) myocardial systolic excursion velocity $\left(S^{\prime}\right)$ is measured at the lateral tricuspid annulus using apical 4-chamber views.

b) tricuspid annular plane systolic excursion (TAPSE) is measured at the lateral annulus using M-mode.

- Aortic, tricuspid, mitral and pulmonary valvular evaluation.

- Atrial electromechanical delay (AEMD) was calculated from colored-TDI recordings. AEMD was determined as the time interval between the beginning of echocardiographic $\mathrm{P}$ wave to the initial of Am-wave (late diastolic wave) in TDI recordings and measured from lateral/tricuspid, lateral/mitral and septal anulus from apical 4-chamber views.

All patients were informed about the study and written consent was obtained for all procedures.

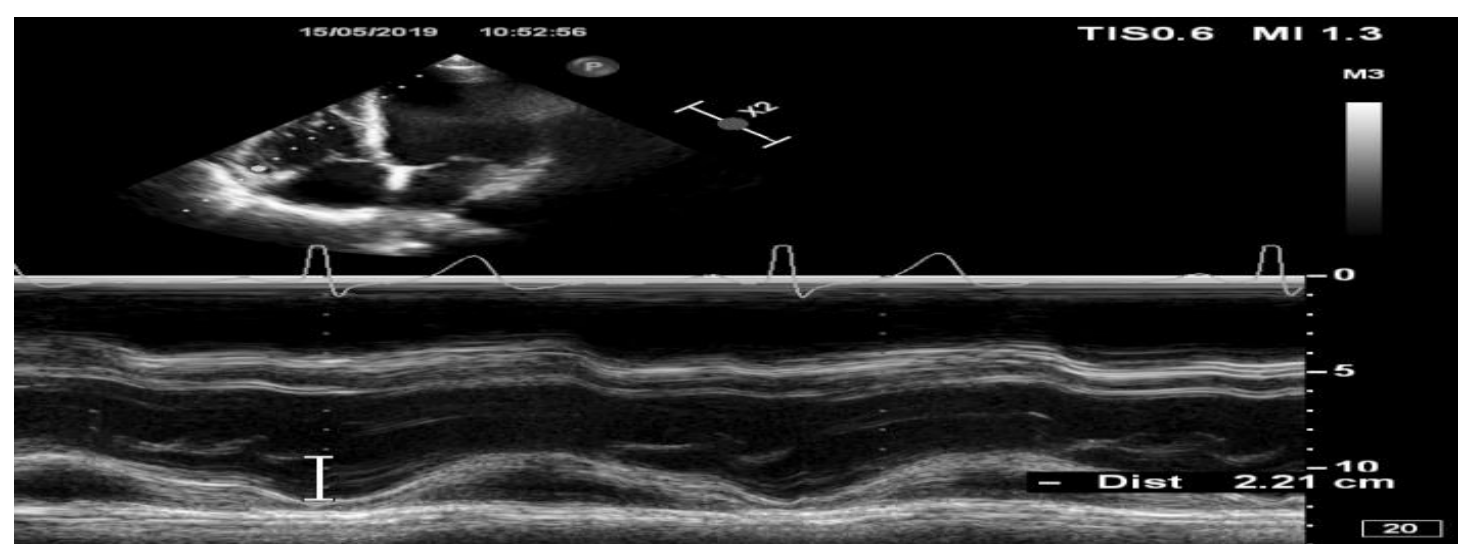

Figure 1. Tricuspid Annular Plane Systolic Excursion (TAPSE) is measured by placing the M-mode cursor through the tricuspid annular plane from apical 4-chamber view.

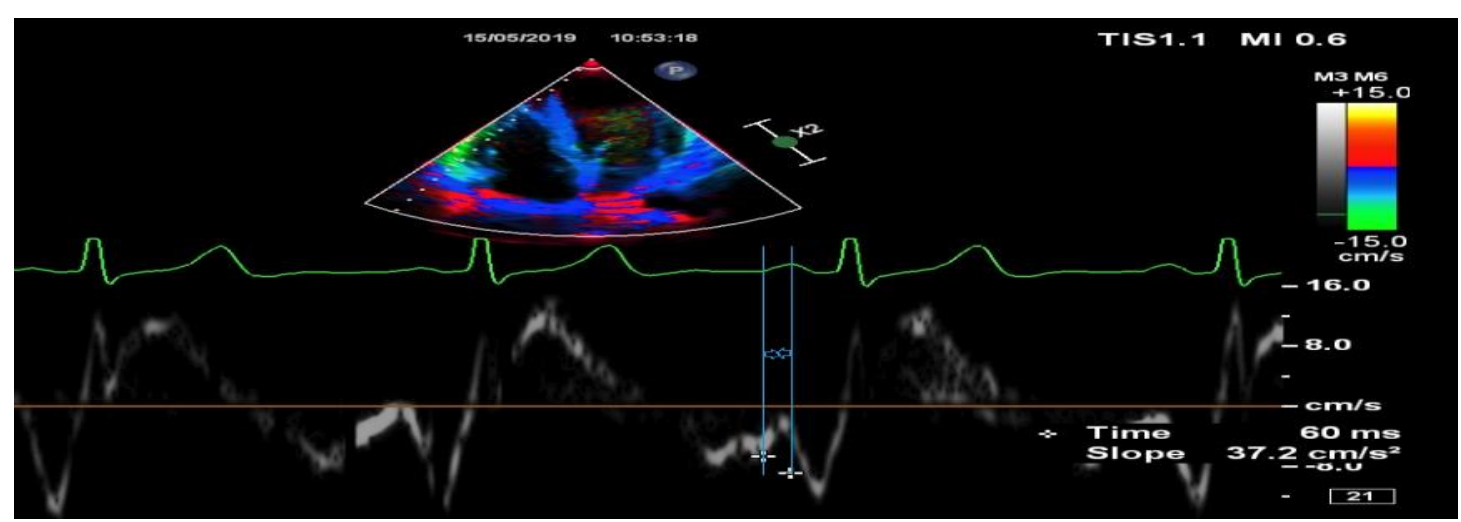

Figure 2. Measurement of the atrial electromechanical delay (AEMD) from lateral tricuspid with tissue Doppler imaging (the interval from the onset of $\mathrm{P}$ wave on the surface ECG to the beginning of the late diastolic wave). 


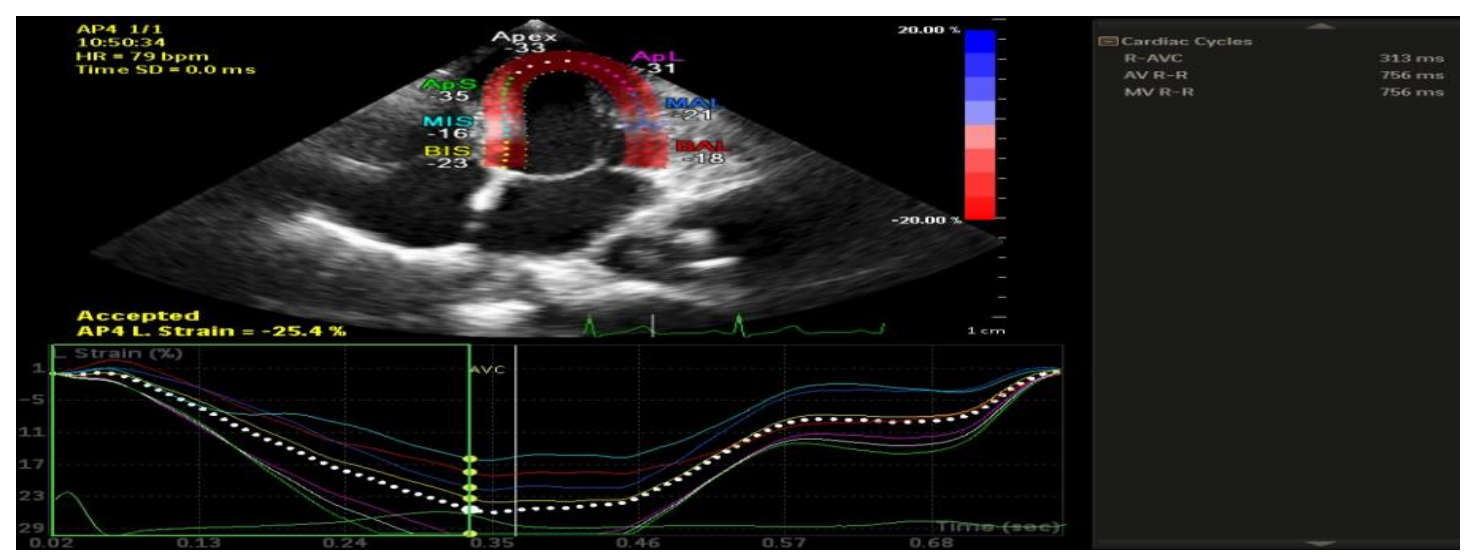

Figure 3. View of the segmental longitudinal strains in apical 4-chamber view during the contraction. At the below, color of each trace corresponds to anatomical points on the 2-D color image to the above. The white dotted line represents average strain.

\section{Statistical Analysis}

All statistical calculations were performed with SPSS 23.0 (SPSS for Windows, Chicago, IL, SA). All categorical variables were expressed percentages $(\%)$ and numerical variables were presented mean \pm standard deviation and mean rank. The normal distribution was determined by histogram and Kolmogorov-Smirnov test. The categorical parameters were compared with Chi-Square test and Fischer's exact test. The continuous variables were analyzed according to normal distribution. Student's t-test was used for normal distribution and the data is not appropriate was evaluated with Mann-Whitney test. The statistical significance level was $\mathrm{p}<0.05$.

\section{Results}

The baseline characteristics of all the patients are given Table 1. The study included 116 female with asthma and 50 control subjects. The mean age of patients with asthma were higher $(\mathrm{p}=0.001)$. The biomass exposure rates were variable between two groups $(\mathrm{p}=0.013)$.

Table 1. Baseline characteristics of control and asthmatic groups

\begin{tabular}{|c|c|c|c|}
\hline Variable & Control $(\mathrm{n}=50)$ & Asthma $(n=116)$ & $P$ value \\
\hline Age, years & $41.58 \pm 13.45$ & $48.91 \pm 12.73$ & $0.001^{\mathrm{T}}$ \\
\hline \multicolumn{4}{|l|}{ Smoking status } \\
\hline Never smoker & $(35) 70 \%$ & $(84) 72.4 \%$ & \multirow{3}{*}{$0.879 \chi^{2}$} \\
\hline Current smoker & (8) $16 \%$ & (19) $16.4 \%$ & \\
\hline Ex smoker & (7) $14 \%$ & (13) $11.2 \%$ & \\
\hline \multicolumn{4}{|l|}{ Biomass exposure } \\
\hline Current exposure & (14) $28 \%$ & (37)31.9\% & \multirow{3}{*}{$0.013 \chi^{2}$} \\
\hline Never exposure & $(28) 56 \%$ & (39) $33.6 \%$ & \\
\hline Previously exposure & (8) $16 \%$ & (40)34.5\% & \\
\hline
\end{tabular}

The comparison of blood parameters are presented in Table 2. $\mathrm{CRP}(\mathrm{p}=0.017), \mathrm{CAR}(\mathrm{p}=0.01)$, eosinophil count were higher and albumin $(\mathrm{p}=0.001)$ was lower in the asthma group. 
Table 2. Blood parameters of control and asthmatic patients

\begin{tabular}{lccc}
\hline Variable & Control $(\mathbf{n = 5 0})$ & Asthma $(\mathbf{n = 1 1 6})$ & P value \\
\hline CRP & $4.12 \pm 9.22$ & $5.42 \pm 10.20$ & $\mathbf{0 . 0 1 7}^{\mathbf{M}}$ \\
Albumin & $46.11 \pm 2.7$ & $44.58 \pm 2.52$ & $\mathbf{0 . 0 0 1}^{\mathbf{T}}$ \\
CAR & $0.09 \pm 0.21$ & $0.12 \pm 0.24$ & $\mathbf{0 . 0 1}^{\mathbf{M}}$ \\
Neutrophil & $79.25_{\mathrm{m}}$ & $79.61_{\mathrm{m}}$ & $0.964^{\mathrm{M}}$ \\
Lymphocyte & $2.21 \pm 0.68$ & $2.39 \pm 0.73$ & $0.136^{\mathrm{T}}$ \\
NLR & $2.44 \pm 1.38$ & $2.25 \pm 1.57$ & $0.425^{\mathrm{M}}$ \\
Platelet & $74.83_{\mathrm{m}}$ & $81.54_{\mathrm{m}}$ & $0.397^{\mathrm{M}}$ \\
PLR & $151.62 \pm 81.16$ & $139.07 \pm 59.76$ & $0.974^{\mathrm{M}}$ \\
Magnesium & $1.98 \pm 0.17$ & $1.99 \pm 0.16$ & $0.982^{\mathrm{T}}$ \\
MPV & $9.99 \pm 1.04$ & $9.95 \pm 1.03$ & $0.834^{\mathrm{T}}$ \\
Eosinophil & $0.12 \pm 0.08$ & $0.21 \pm 0.19$ & $\mathbf{0 . 0 0 1}^{\mathbf{M}}$ \\
25-hydroxyvitamin D & $13.58 \pm 7.76$ & $12.38 \pm 5.46$ & $0.819^{\mathrm{M}}$ \\
PCT & $0.30 \pm 0.092$ & $0.30 \pm 0.05$ & $0.528^{\mathrm{M}}$ \\
PDW & $16 \pm 0.34$ & $15.98 \pm 0.29$ & $0.864^{\mathrm{M}}$
\end{tabular}

Data expressed as mean \pm standard deviation. CRP, c-reactive protein; CAR, c-reactive protein/albumin ratio; NLR, neutrophil/lymphocyte ratio; PLR, platelet/lymphocyte ratio; MPV, mean platelet volume; PCT, plateletcrit; PDW, platelet distribution width, m, mean rank; M, Mann Whitney U; ${ }^{\mathrm{T}}$, t-Student test.

In the present study, conventional and tissue echocardiographic parameters in healthy and asthmatic females were assessed. Echocardiographic findings of left heart and atrial conduction times in two groups are given Table 3. End-systolic diameter $(\mathrm{p}=0.029)$, interventricular septum thickness $(\mathrm{p}=0.022)$ and posterior wall thickness $(\mathrm{p}=0.027)$ were higher in the group with asthma. LV diastolic functions including Emax mitral $(\mathrm{p}=0.040)$ and $\mathrm{E} / \mathrm{A}$ mitral decreased in asthmatic females. Aortic diameters consist of sinus $(\mathrm{p}=0.041)$, sinutubular junction $(\mathrm{p}=0.037)$ and ascending aorta $(\mathrm{p}=0.006)$ increased significantly and lateral/tricuspid AEMD prolonged $(\mathrm{p}=0.038)$ in the patients with asthma. 
International Journal of Scientific and Technological Research

ISSN 2422-8702 (Online), DOI: 10.7176/JSTR/6-03-06

Special Issue of Health Sciences, Vol.6, No.3, 2020

ISTE

Table 3. Echocardiographic findings of left heart and atrial conduction times in control and asthmatic patients

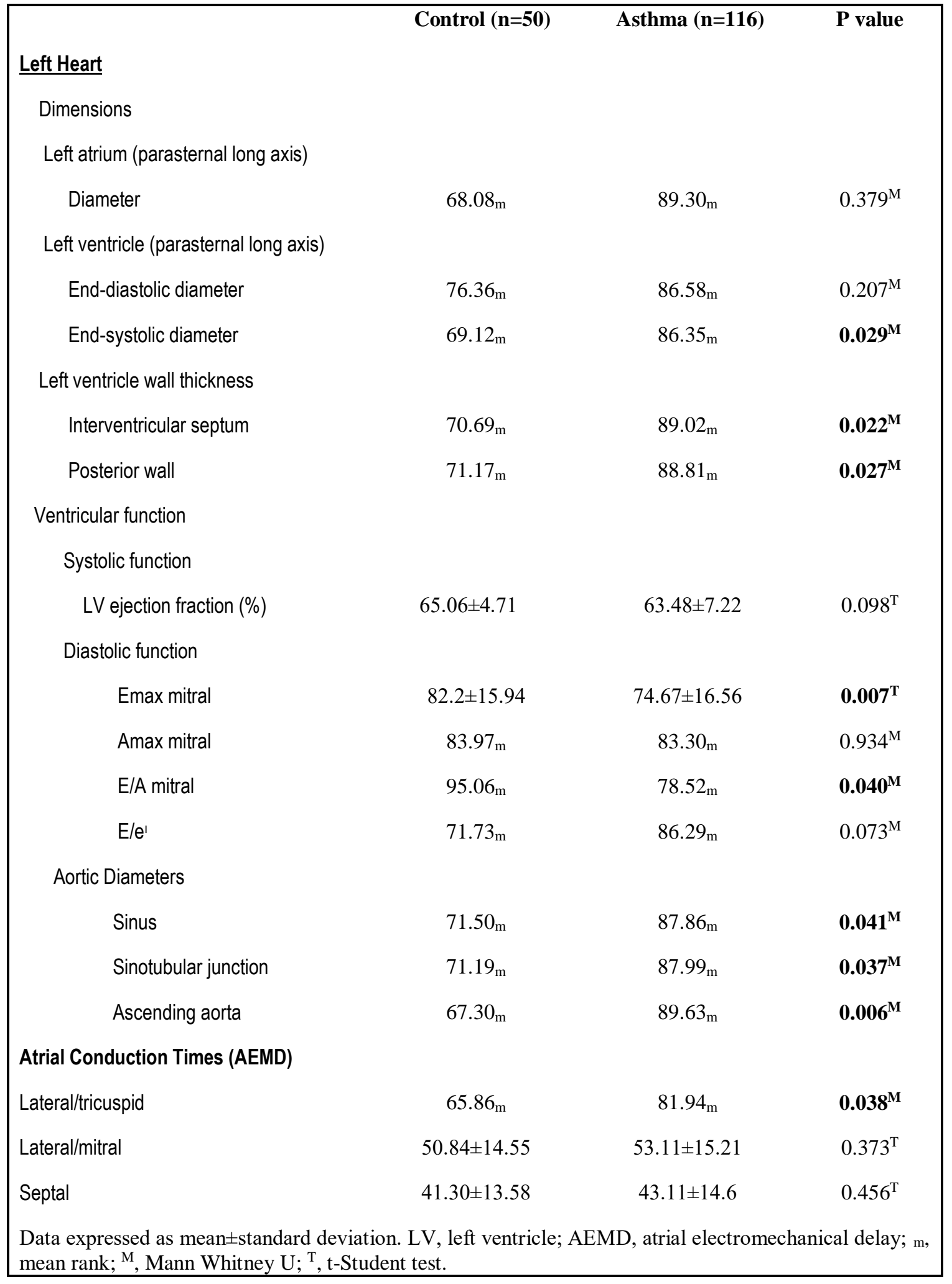

Echocardiographic assessment of right right are presented in Table 4. In the asthma group, RV free wall thickness was higher $(\mathrm{p}=0.043)$. 
Table 4. Echocardiographic findings of right heart in control and asthmatic patients

\begin{tabular}{|c|c|c|c|}
\hline & Control $(\mathrm{n}=50)$ & Asthma (n=116) & $P$ value \\
\hline \multicolumn{4}{|l|}{$\underline{\text { Right Heart }}$} \\
\hline \multicolumn{4}{|l|}{ Dimensions } \\
\hline \multicolumn{4}{|l|}{ Right ventricle } \\
\hline Basal & $81.88_{\mathrm{m}}$ & $84.20_{\mathrm{m}}$ & $0.774^{\mathrm{M}}$ \\
\hline Mid & $83.11_{\mathrm{m}}$ & $83.67 \mathrm{~m}$ & $0.945^{\mathrm{M}}$ \\
\hline Vertical & $83.96_{\mathrm{m}}$ & $83.30_{\mathrm{m}}$ & $0.935^{\mathrm{M}}$ \\
\hline \multicolumn{4}{|l|}{ RV systolic function } \\
\hline $\mathrm{S}^{\prime}$ & $88.38 \mathrm{~m}$ & $81.40_{\mathrm{m}}$ & $0.390^{\mathrm{M}}$ \\
\hline TAPSE & $88.32_{\mathrm{m}}$ & $79.95_{m}$ & $0.296^{\mathrm{M}}$ \\
\hline RV free wall thickness & $71.20_{\mathrm{m}}$ & $87.46_{\mathrm{m}}$ & $0.043^{M}$ \\
\hline Systolic pulmonary arterial pressure $(\mathrm{mmHg})$ & $82.07 \mathrm{~m}$ & $82.69 \mathrm{~m}$ & $0.936^{\mathrm{M}}$ \\
\hline
\end{tabular}

\section{Discussion}

This study confirms that CRP, CAR, eosinophil count were higher and albumin was lower in the asthma group. Several studies have recently reported that increased inflammatory markers and cells including CRP, CAR and eosinophil are associated with chronic inflammatory lung disease. ${ }^{9-11}$ Asthmatic inflammatory cells may increase reactive oxygen species and damage of those can be prevented with antioxidant agents. Albumin is an acute phase reactant and is also defined non-enzymatic antioxidant agent due to binding of oxidation-reduction active metals. Vural et al. found that albumin level decreased in patients with broncial asthma. ${ }^{9}$ The patients were in stable period, but these levels were determined because low-grade chronic inflammation.

In the current study, LV end-systolic diameter, interventricular thickness and posterior wall thickness were higher in the asthmatic groups and diastolic functions including Emax mitral and E/A mitral impaired. Supraventricular tachycardias that during the exacerbation period of asthmatic patients my lead to changes in diameters, septal hypertrophy, LV dysfuntion and increased pulmonary artery pressure. In studies by De-Paulo, Ozkan and Abdalla et al., similar results were found in the diastolic functions with a decrease of peak velocity blood flow in early diastole and increase of peak velocity flow in the late diastole. ${ }^{1,2,5}$

We found that aortic diameters including sinus, sinutubular junction and ascending aorta increased significantly. The pathophysiology of aneurysmatic dilatation of the aorta and asthma is similar and inflammation is responsible for formation. It is determined that macrophages, lymphocytes and neutrophil, mast cells, immunglobulin E increase in the aneurysmatic arterial wall. In asthmatic patients, leukotriene $\mathrm{B}_{4}$ leads to translocation of inflammatory cells to the alveoli and bronchi and leukotriene $\mathrm{C}_{4}$, $\mathrm{D}_{4}$ and $\mathrm{E}_{4}$ cause increase of vascular permeability and contraction of smooth muscle cell induces bronchoconstruction. Increment of leukotriene $\mathrm{B}_{4}$ levels in asthmatic patients may increase aortic wall inflammatory cell aggreation. ${ }^{12}$

In the study, lateral/tricuspid AEMD prolonged in the asthmatic females. Asthma disease may cause electrocardiographic abnormalities including atrial fibrillation, sinus tachycardia, bradycardia and right 
axis deviation. The electrophysiological abnormalities of the atrium may cause the possibility of atrial fibrillation occurence. Inflammation, decreased oxygenation, pulmonary hypertension, diastolic function impairment, oxidative stress, changes of atrial size leading to deformation may cause atrial conduction abnormalities including prolongation of atrial conduction times in patients with obstructive pulmonary disease. ${ }^{13,14}$

In the present study, RV free wall thickness informing that RV systolic function was higher in asthmatic group. In several studies by Ghandi, Ghaderian and Shedeed et al., similar results were found that RV wall thickness significantly different between asthmatic patients and healthy subjects. ${ }^{13,15,16}$ In contrast, Ozkan et al. found no significant differences in this variable between two groups. ${ }^{1}$

\section{Limitations of the study}

The main limitations of this study were the planning of the study with only women, the lack of detailed functional capacity of the lung and the inadequate implementation of cardiopulmonary stress tests.

\section{Conclusion}

The study showed that LV end-systolic diameter, LV wall thickness, LV diastolic functions including Emax, E/A, aortic diameters including sinus, sinutubular junction, ascending aorta, lateral/tricuspid AEMD and RV systolic function including RV free wall thickness were affected in females in asthma.

\section{References}

1. Ozkan EA, Khosroshahi HE. Evaluation of the left and right ventricular systolic and diastolic function in asthmatic children. BMC Cardiovasc Disord 2016;16:145.

2. Abdalla ME, El Azeem HA. Echocardiographic evaluation of ventricular function in young adults with bronchial asthma. Egypt J Chest Dis Tuberc 2013;62(1):27-31.

3. Perincek G, Avcı S. Factors affecting the hospitalization of female patients with asthma. J Surg Med 2019;3(9):698-701.

4. Keet CA, McCormack M. Cardiac asthma: an old term that may have new meaning? J Allergy Clin Immunol Pract 2016;4:924-5.

5. De-Paula CR, Magalhães GS, Jentzsch NS, Botelho CF, Coelho Mota CC, Murça TM, et al. Echocardiographic assessment of ventricular function in young patients with asthma. Arq Bras Cardiol 2018; 110(3):231-9.

6. Ruiz-Bailén M, Cobo-Molinos J, Espada-Fuentes JC , Castillo-Rivera AM, Martínez-Ramírez MJ, Cárdenas-Cruz A. The acute asthma: it's just a respiratory disease? A speckle tracking echocardiography study. J Clin Respir Dis Care 2016;2(4):122.

7. Highley AD, Cookman C, Morrow LE, Malesker MA. Severe asthma: An update for 2019. US Pharm 2019;44(7):2-7.

8. Lang RM, Badano LP, Mor-Avi V, Afilalo J, Armstrong A, Laura Ernande L, et al. Recommendations for cardiac chamber quantification by echocardiography in adults: an update from the American Society of Echocardiography and the European Association of Cardiovascular Imaging. J Am Soc Echocardiogr 2015;28:1-39.

9. Vural H, Uzun K. Serum and red blood cell antioxidant status in patients with bronchial asthma. Can Respir J 2000;7(6):476-80.

10. Halvani A, Tahghighi F, Nadooshan HH. Evaluation of correlation between airway and serum inflammatory markers in asthmatic patients. Lung India 2012; 29(2): 143-6.

11.Ventura JC, Hauschild DB, Moreira EAM, Pereira LCR, Rosa AF, Barbosa E, et al. C-reactive protein/albumin ratio is associated with lung function among children/adolescents with cystic fibrosis: a three-year longitudinal study. Sao Paulo Med J 2018; 136(1):29-36. 
12. Liu CL, Wemmelund H, Wang Y, Liao M, Lindholt S, Johnsen SP, et al. Asthma associates with human abdominal aortic aneurysm and rupture. Arterioscler Thromb Vasc Biol 2016; 36(3): 5708.

13. Ghandi Y, Habibi D, Abas M. The effect of bronchial asthma on interatrial electromechanical delay coupling obtained using tissue Doppler Imaging. Iran J Med Sci 2019;44(3):196-203.

14. Goudis CA. Chronic obstructive pulmonary disease and atrial fibrillation: An unknown relationship. J Cardiol 2017;69:699-705.

15. Ghaderian M, Sayedi SJ, Momen T, Zandi Z, Reisi M. Evaluation of right ventricular function by tissue Doppler Echocardiography in asthmatic children. Int J Pediatr 2016;4(11):3941-8.

16. Shedeed S. Right ventricular function in children with bronchial asthma: a tissue Doppler echocardiographic study. HMJ 2010;4(1):80-85. 